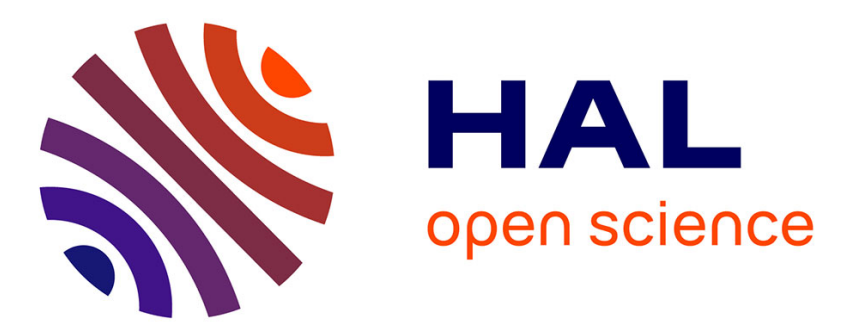

\title{
Water structure, dynamics and sum-frequency generation spectra at electrified graphene interfaces
}

Yiwei Zhang, Hilton B de Aguiar, James T Hynes, Damien Laage

\section{To cite this version:}

Yiwei Zhang, Hilton B de Aguiar, James T Hynes, Damien Laage. Water structure, dynamics and sum-frequency generation spectra at electrified graphene interfaces. Journal of Physical Chemistry Letters, 2020. hal-03024612

\section{HAL Id: hal-03024612 https://hal.science/hal-03024612}

Submitted on 25 Nov 2020

HAL is a multi-disciplinary open access archive for the deposit and dissemination of scientific research documents, whether they are published or not. The documents may come from teaching and research institutions in France or abroad, or from public or private research centers.
L'archive ouverte pluridisciplinaire HAL, est destinée au dépôt et à la diffusion de documents scientifiques de niveau recherche, publiés ou non, émanant des établissements d'enseignement et de recherche français ou étrangers, des laboratoires publics ou privés. 


\title{
Water structure, dynamics and sum-frequency generation spectra at electrified graphene interfaces
}

\author{
Yiwei Zhang, ${ }^{\dagger}$ Hilton B. de Aguiar, ${ }^{\ddagger}$ James T. Hynes, ${ }^{\dagger} \uparrow$ and Damien Laage, ${ }^{*} \dagger$ \\ $\dagger$ PASTEUR, Department of Chemistry, École normale supérieure, PSL University, \\ Sorbonne Université, CNRS, 75005 Paris, France \\ $\ddagger$ Département de Physique, École normale supérieure, PSL University, CNRS, 24 rue \\ Lhomond, 75005 Paris, France \\ \Department of Chemistry, University of Colorado, Boulder, CO 80309, USA \\ E-mail: damien.laage@ens.fr
}




\begin{abstract}
The properties of water at an electrified graphene electrode are studied via classical molecular dynamics simulations with a constant potential approach. We show that the value of the applied electrode potential has dramatic effects on the structure and dynamics of interfacial water molecules. While a positive potential slows down the reorientational and translational dynamics of water, an increasing negative potential first accelerates the interfacial water dynamics before a deceleration at very large magnitude potential values. Further, our spectroscopic calculations indicate that the water rearrangements induced by electrified interfaces can be probed experimentally. In particular, the calculated water vibrational sum-frequency generation (SFG) spectra shows that SFG specifically reports on the first two water layers at $0 \mathrm{~V}$, but that at larger magnitude applied potentials the resulting static field induces long-range contributions to the spectrum. Electrified graphene interfaces provide promising paradigm systems for comprehending both short- and long-range neighboring aqueous system impacts.
\end{abstract}

\title{
Graphical TOC Entry
}

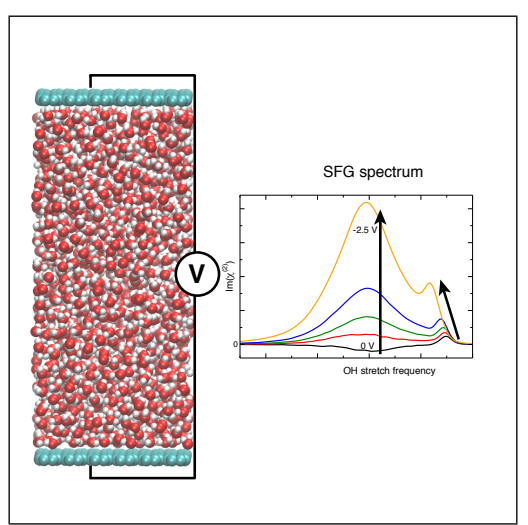


The first layer (or layers) of water molecules at the interface with an electrified material plays a central role in a broad range of processes of fundamental and applied interest, including electrochemical reactivity, ${ }^{1}$ electrocatalysis, corrosion and water desalination. ${ }^{2}$ Accordingly, a detailed molecular characterization of an electrified interface's impact on the structure and dynamics of this water layer is of considerable interest. The graphene/water interface is a particularly attractive paradigm system in this connection, due to the great practical relevance of carbon/water interfaces ${ }^{2}$ and to several appealing features of graphene: graphene exhibits a well-defined atomically flat surface, ${ }^{3}$ it can be electrified due to its semimetallic character, ${ }^{4}$ and it is transparent in the relevant infrared frequency range, making the buried water/graphene interface accessible to surface-specific vibrational spectroscopy experiments. ${ }^{5}$ Here we show how molecular dynamics simulations and sum-frequency generation (SFG) spectra calculations reveal the dramatic effects of applied electric potentials on the structural and dynamical properties of interfacial water layers. In the following, we first describe our simulation methodology, then analyze how the electrode potential changes the structure and the dynamics of the interfacial layers, and finally show how the electrode impacts on water can be probed with SFG spectroscopy.

Our system comprises a slab of $1158 \mathrm{SPC} / \mathrm{E}$ water molecules between two rigid single graphene sheets, each containing 240 carbon atoms. The SPC/E potential was shown to provide an excellent description of water dynamics at ambient conditions. ${ }^{6}$ In typical SFG experiments ${ }^{7}$ graphene is deposited on a solid substrate and can thus be approximated as immobile. The simulation box is periodically replicated in the two directions parallel to the graphene sheets. Carbon-water non-bonding interactions are described by a LennardJones potential whose parameters were optimized in ref 8 to reproduce the results of DFTbased molecular dynamics (MD) simulations; such an MD approach allows the necessary extensive and time-extended simulations. Long-range Coulomb interactions are described with a particle-mesh Ewald approach, adapted to the slab geometry. Simulations are run at a series of electrode potentials ranging from $0 \mathrm{~V}$ to $\pm 2.5 \mathrm{~V}$ (potentials of at least $\pm 1.5 \mathrm{~V}$ 
are experimentally accessible ${ }^{9}$ and the largest $\pm 2.5 \mathrm{~V}$ potentials are used here to highlight the trends). Graphene carbon atom charges are determined using the constant potential method, ${ }^{10,11}$ in which each carbon atom bears a Gaussian charge of inverse width $1.979 \AA^{-1}$, with the charge amplitude determined at each time step such that the potential on each electrode is held fixed; this method has already been successfully employed to describe a broad range of electrified interfaces. ${ }^{10-14}$ Each trajectory is first equilibrated in the presence of a $298 \mathrm{~K}$ thermostat, and subsequently propagated for $4 \mathrm{~ns}$ in the microcanonical ensemble using the LAMMPS software, ${ }^{15}$ where the constant potential method was implemented. ${ }^{14}$ In what follows, all reported error bars provide the $95 \%$ confidence intervals determined from block calculations on independent 400 ps (resp. 50 ps) intervals for the structural and dynamical properties (resp. SFG spectra).

We first characterize the effects of increasing electrode potentials on the liquid water structure. These results - which prove to be quite valuable for comprehending the water dynamics and SFG spectra - show that liquid translational and orientational responses at the positive and negative electrodes are in stark contrast. Figure 1a reports the changes in the density profile across the cell (see also Fig. S1 for the $\mathrm{O}$ and $\mathrm{H}$ atom contributions). We first note that at both interfaces, the presence of an electrode at $0 \mathrm{~V}$ potential does not noticeably affect the water density profile beyond the first two layers (i.e. $\approx 7 \AA$ from the electrode). However, as soon as a potential difference is applied between the two graphene sheets, the hydration layer densities change in a very different fashion at the two interfaces. At the positive electrode, a growing potential leads predominantly to a more pronounced structuring of the first two layers, with higher and sharper first two density maxima and a lower first density minimum, and with a slight shift of the first density maximum towards the graphene sheet. In contrast, at the negative electrode a more complex restructuring of the first layer occurs, with a progressive splitting of the first layer.

We now embark on an explanation of these observations, first focusing on the water molecule orientations within the first layer, whose boundary is here defined by the first 
minimum in the density profile. We start with the reference situation at $0 \mathrm{~V}$ potential (which is trivially the point of zero charge due to the graphene's apolar nature). Figure 1b (black line) shows the probability distribution of the angle $\theta$ between the $\mathrm{OH}$ groups of water molecules within the first layer and the vector normal to the graphene sheet pointing towards graphene. Since the average charge on each carbon atom is 0 , the graphene sheets behave as extended apolar, hydrophobic interfaces. Interfacial water molecules thus tend to form hydrogen-bonds with neighboring water molecules, while the flat-interface-imposed geometric constraints force the water hydrogen-bond network to sacrifice some hydrogen-bonds, leading to a small fraction of dangling $\mathrm{OH}$ groups pointing towards the interface. ${ }^{16}$ In contrast to the angular distribution that would be expected in the isotropic, uniform case (dashes), the distribution shows that most water $\mathrm{OH}$ groups lie almost tangent to the interface $\left(\theta \simeq 80^{\circ}\right.$, see inset in Fig 1b); another population is oriented away from graphene towards the bulk to form hydrogen-bonds with second-layer water molecules $\left(\theta>140^{\circ}\right.$, see inset in Fig 1b), and finally a small fraction of $\mathrm{OH}$ groups are in a 'dangling' situation, oriented towards the interface $\left(\theta<40^{\circ}\right.$, see inset in Fig 1b). This interfacial water molecular arrangement is very similar to that found e.g. at the air/water interface and at extended hydrophobic interfaces ${ }^{17,18}$ (see SI); it is also consistent with prior simulation results obtained with both classical and DFT-based molecular dynamics ${ }^{8,19-21}$ of water at an uncharged graphene interface.

We now enquire as to the rearrangements induced by applying electrode potentials, starting with negative potentials. Figure $1 \mathrm{~b}$ shows that the growing negative charge on the carbon atoms favors $\mathrm{OH}$ group orientations toward the interface (i.e. dangling $\mathrm{OH}$ ), with a concomitant depletion in the populations of both $\mathrm{OH}$ groups tangent to the interface and those pointing towards bulk water. The splitting of the density profile's first peak in Fig 1a thus arises from the appearance of a new population of water molecules directly interacting with graphene via one of their $\mathrm{OH}$ groups, in a fashion similar to a donated hydrogen-bond. This population is therefore closer to the graphene surface, while the other water molecules within the first layer do not form such a hydrogen-bond and are thus slightly farther from 
that surface (see Fig. S1).

The situation is quite different at the positive electrode interface: Figure 1c shows that increasing potentials only induce minor changes in the water molecule orientations. While the most favorable $\mathrm{OH}$ orientation remains that tangent to the interface, the main change induced by the positive electrode potential is the disfavored $\mathrm{OH}$ groups' orientations towards the interface caused by the growing carbon positive charges.

This structural analysis has emphasized the strong asymmetry in the water response in the presence of positive and negative electrode potentials. It is important to note that such a result cannot be understood via a simplified point dipole representation of water molecules. But this inadequacy is not surprising, since for first layer water molecules, the molecular size and especially the distance between the oxygen and hydrogen charges cannot be assumed to be much shorter than the water-electrode distance. Indeed, such asymmetry is also found e.g. in the hydration structures of cations and anions, and in the hydration free energies of cations and anions of similar sizes. ${ }^{22}$ In contrast, this asymmetry is well described with the distributed point charge water model used in classical MD.

We now turn to the study of the reorientational and translational dynamics for the water molecules within the first layer at the graphene interface. Our first focus is on the $\mathrm{OH}$ group reorientation time correlation function ${ }^{23}$ (tcf) relevant for ultrafast infrared anisotropy and NMR measurements, ${ }^{23}$

$$
C_{2}^{\mathrm{OH}}(t)=\left\langle P_{2}\left[\mathbf{u}_{\mathrm{OH}}(0) \cdot \mathbf{u}_{\mathrm{OH}}(t)\right]\right\rangle
$$

where $\mathbf{u}_{\mathrm{OH}}(\mathbf{t})$ is the unit vector along an $\mathrm{OH}$ group's direction, $P_{2}$ is the second-order Legendre polynomial, and the $\langle\ldots\rangle$ ensemble average is performed over all water $\mathrm{OH}$ groups within the first layer at $t=0$. The reorientation time $\tau_{2}^{\mathrm{OH}}$ is the long-time decay time of this reorientation time correlation function. ${ }^{23}$ Our results in Fig $2 \mathrm{a}$ show that the impact of an electrified interface on water reorientational dynamics differs dramatically for positively and negatively charged electrodes. While water reorientation is markedly slowed by an increasing positive electrode potential, it exhibits a surprising acceleration next to negatively charged 


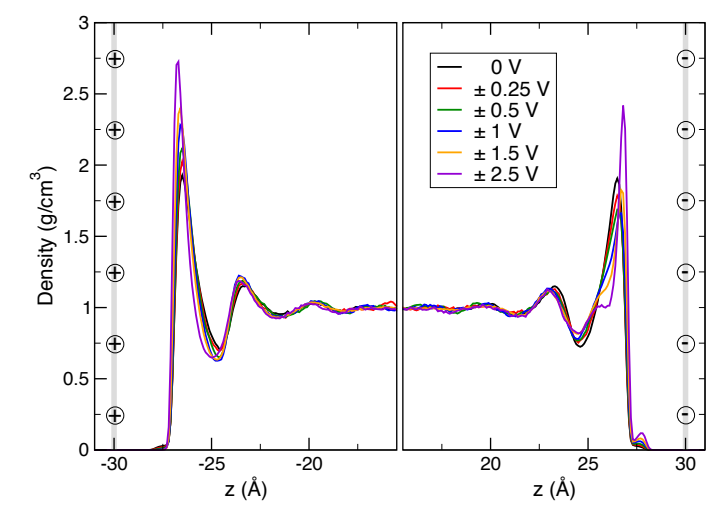

b

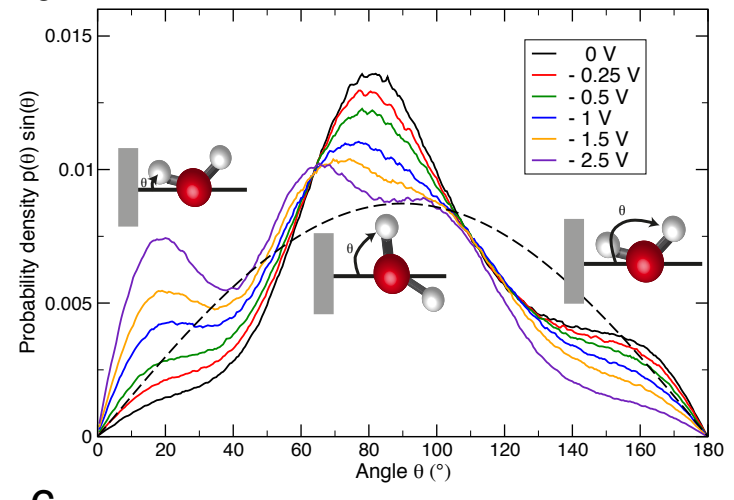

C

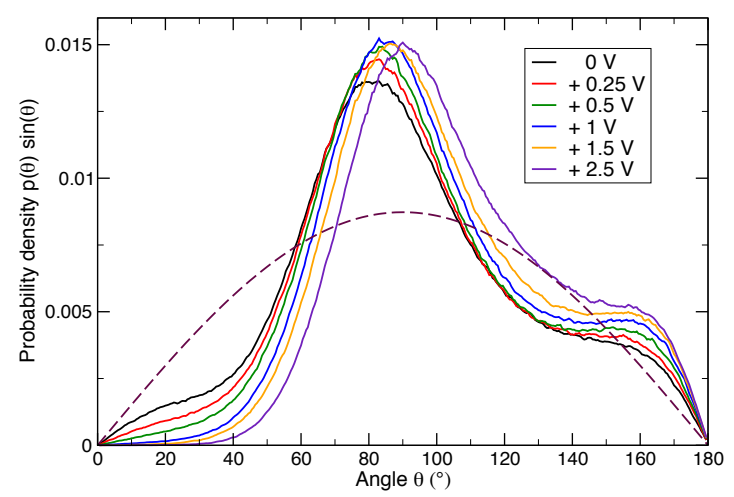

Figure 1: Interfacial water structural properties. a) Water density profile along the $z$ axis, with the positive interface at $z=-30 \AA$ and the negative interface at $z=+30 \AA$. b) angular probability density of water $\mathrm{OH}$ groups within the first layer at the negative interface, $\mathrm{c}$ ) idem at the positive interface. In each case $\theta$ is defined as the angle between the OH group and the vector normal to the interface and pointing towards graphene (see insets in panel b). The dashed lines show the isotropic probability situation.

electrodes. We further find that, in contrast to the monotonic reorientation slowdown for increasing positive potentials, for negative potentials $\tau_{2}^{\mathrm{OH}}$ exhibits an initial acceleration of 
the reorientation, followed by an extremum in the neighborhood of $-1.5 \mathrm{~V}$ where $\tau_{2}^{\mathrm{OH}}$ is smallest and close to the bulk water value; more negative electrode potentials then lead to reorientational slowdown (our key point here is the existence - not the precise location - of this extremum, supported by the clear non-monotonic change in $\tau_{2}^{\mathrm{OH}}$ between $0 \mathrm{~V}$ and -2.5 $\mathrm{V})$.
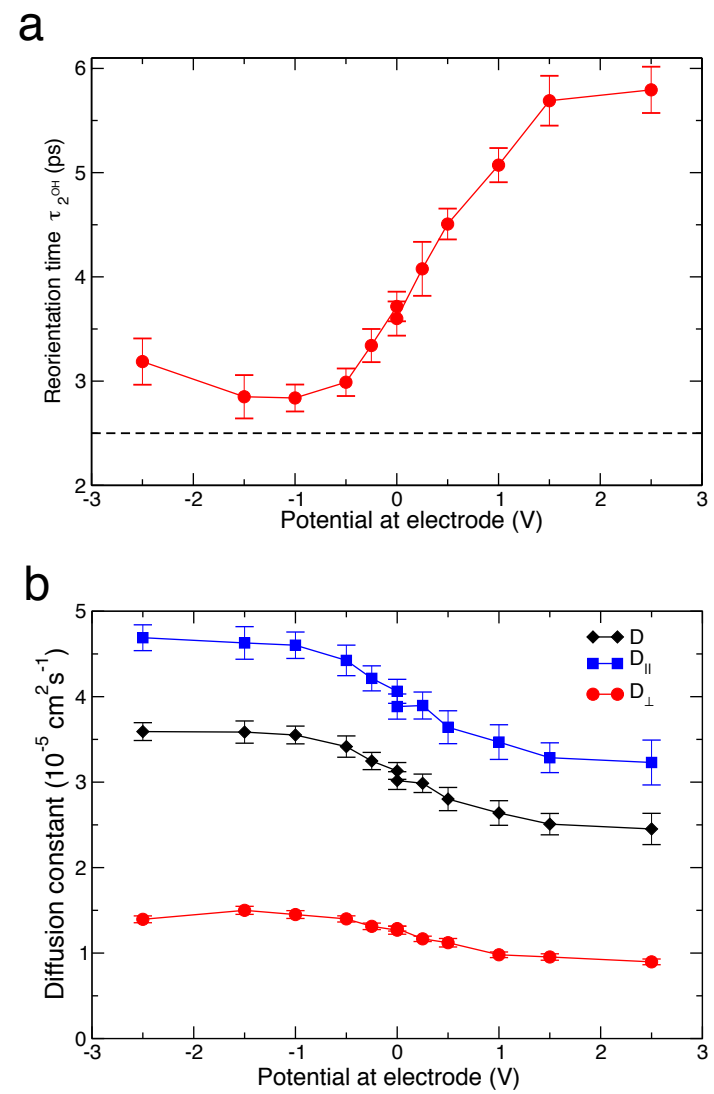

Figure 2: Interfacial water dynamical properties. a) Reorientation time $\tau_{2}^{\mathrm{OH}}$ eq 1 for water $\mathrm{OH}$ groups initially within the interfacial layer at a series of electrode potentials, determined from an exponential fit of $C_{2}^{\mathrm{OH}}$ eq 1 on the 2-10 ps time interval; b) Translational diffusion constant of interfacial water molecules, together with its components parallel and perpendicular to the interface, determined from the mean square displacement of water molecules initially in the first layer via a linear fit over the $2-10$ ps interval.

Water translational dynamics at the interface exhibits the same type of opposite responses to positive and negative electrode potentials just described for reorientation (Fig $2 \mathrm{~b}$ ). It is seen that translational diffusion at the interface is accelerated by negative electrode potentials 
but slowed by positive potentials. Examination of the components of the displacement parallel and perpendicular to the graphene sheet (Fig $2 \mathrm{~b}$ ) reveals both to be affected in a qualitatively similar fashion. Finally, an analogue of the extremum in the reorientational dynamics at approximately $-1.5 \mathrm{~V}$ can be found for the translational dynamics orthogonal to the interface, but not for that along the plane.

Some of us established ${ }^{24}$ that sudden, large amplitude angular jumps between hydrogenbond acceptors are the main reorientation pathway for water molecules. These jumps are also intimately connected to the translational dynamics since translational displacements beyond the water molecule's initial solvent cage require hydrogen-bond exchanges. The extended jump model and its subsequent developments have been shown to provide an insightful and almost quantitative description of the impact of a wide variety of different local environments on the hydrogen-bond jump dynamics and thus on the water reorientation time. ${ }^{23}$

As we now argue for water at electrified interfaces, the jump picture again provides an intuitive and molecular understanding of the surprisingly different behaviors at positive and negative electrodes. The key difference is that positive interfaces act as H-bond donors while negative interfaces act as H-bond acceptors, resulting in different effects on the H-bond jumps. ${ }^{25}$ At positive electrodes, our results in Figs 1a,c show that increasing electrode potentials do not qualitatively change the water molecules' arrangement (i.e. the locations of the density extrema) but produce a more pronounced structuring (i.e. a change in the extrema amplitudes). Prior work by some of us established ${ }^{26}$ that such structural enhancements including e.g. higher first maximum and lower first minimum in the water-water radial distribution function - hinder the rearrangements necessary for the hydrogen-bond jumps, and that these can be quantitatively related to a slowing down of the jump dynamics, and thus of the reorientation dynamics.

As for the negative electrode case, the non-monotonic change in the reorientation dynamics with growing negative charges on the graphene interface seen in Fig $2 \mathrm{a}$ is very similar to that observed ${ }^{27}$ for the dynamics of water next to a silica surface whose hydrophilicity 
was changed by progressively switching the surface's atomic charges from a hydrophobic limit of zero up to their full values. The reorientation time's non-monotonic behavior was shown ${ }^{27}$ to be due to a combination of two effects: a change in the populations of $\mathrm{OH}$ groups pointing towards the interface and tangentially to the interface, and the slowing down in the jump dynamics of $\mathrm{OH}$ groups directed towards the interface when the surface charges are increased, strengthening the water/interface attractive interaction. We anticipate the same underlying mechanism to be responsible for the negative electrode potential's effect on the interfacial dynamics of water. Starting from $0 \mathrm{~V}$, decreasing the electrode potential increases the fraction of $\mathrm{OH}$ groups pointing towards graphene, and at low potential the water-graphene interaction remains weak, so that these dangling $\mathrm{OH}$ groups reorient rapidly; the average water reorientation time thus decreases. When the electrode potential is very negative, the graphene-water interaction is stronger; the jump time accordingly increases, thus slowing down the average reorientation dynamics, and explaining the extremum at intermediate negative potentials. The change in interfacial water dynamics here would originate from the interface's impact on the hydrogen-bond jumps, just as was previously found for a broad range of interfaces. ${ }^{27,28}$

We now examine whether the interfacial water layer's structural rearrangements resulting from different electrode potentials that we have described can be probed experimentally. Vibrational sum-frequency generation (SFG) suggests itself as a tool, since it has been successfully used to characterize water molecules at a range of aqueous interfaces, including air/water, alkane/water and silica/water interfaces. ${ }^{29-31}$ The buried interface between an aqueous solution and an electrochemical material presents a considerable challenge, ${ }^{32-34}$ but graphene is a promising system here, since it combines an atomically well-defined surface ${ }^{3}$ and IR transparency in the $\mathrm{OH}$ stretch region. ${ }^{5}$ Recent experimental results are encouraging: SFG spectra at an uncharged graphene/water interface have been measured, ${ }^{5}$ and a successful implementation in an electrochemical cell has been reported, ${ }^{7}$ although experimental challenges to prevent water intercalation between substrate and graphene remain. 
We pursue SFG's applicability here by calculating the resonant susceptibility accessible in SFG measurements with heterodyne detection, ${ }^{30,31,35}$

$$
\chi_{p q r}^{(2)}(\omega)=\frac{i \omega}{k_{B} T} \int_{0}^{\infty} \mathrm{d} t e^{i \omega t}\left\langle\alpha_{p q}(t) \mu_{r}(0)\right\rangle
$$

where $\alpha$ is the water polarizability tensor, $\mu$ is the dipole moment, and the $p q r$ indices designate the tensor elements in the $x y z$ space-fixed coordinate frame. In what follows we focus on the $s s p$ polarization scheme, i.e. $p q r=y y z$, since its interpretation is the most straightforward. The water molecular polarizability and dipole are determined at every step of the simulation using the empirical map developed in ref 36 connecting these quantities to the local electric field experienced by the water $\mathrm{H}$ atom along the $\mathrm{OH}$ axis. This method was successfully applied to the air/water SFG spectra. ${ }^{36}$ We follow the same approach to calculate the spectrum that would be measured using a dilute HOD in liquid $\mathrm{D}_{2} \mathrm{O}$ solution; this isotopic mixture allows probing of a local $\mathrm{OH}$ mode, decoupled from the other OD stretches in the liquid, including the same water molecule's OD group. In order to separately determine the SFG spectra at each of the system's two interfaces, we use a switching function centered on the simulation box's middle and based on the oxygen atom position, following recent suggestions. ${ }^{37,38}$

The resulting SFG spectrum for water at the graphene interface at $0 \mathrm{~V}$ (Fig 3a) is generally typical of those already found at air/water interfaces. ${ }^{30,31,35,36,38}$ It exhibits two main bands, a high-frequency $\simeq 3700 \mathrm{~cm}^{-1}$ positive band and a lower-frequency $\simeq 3400 \mathrm{~cm}^{-1}$ negative band. The positive band arises from $\mathrm{OH}$ groups which are pointing towards the interface with graphene, and its high frequency implies that these $\mathrm{OH}$ groups are weakly or not hydrogen-bonded. (At the air/water interface, this band was shown to arise from 'dangling' OH groups at the surface. ${ }^{16}$ ) Regarding the lower frequency band, its sign implies that it originates from water $\mathrm{OH}$ groups pointing away from the interface, towards the bulk liquid, and its average frequency shows that these $\mathrm{OH}$ groups are engaged in hydrogen bonds. 
The calculated $0 \mathrm{~V}$ SFG spectrum is thus consistent with the angular distribution shown in Fig 1b, but it is mostly sensitive to $\mathrm{OH}$ groups pointing towards and away from the graphene-water interface; the dominant tangent $\mathrm{OH}$ groups population does not lead to an SFG signal for the chosen ssp polarization.

Our present spectrum is in excellent agreement with that recently obtained by DFT-based molecular dynamics simulations at a graphene interface with no applied potential ${ }^{39}$ (the OD stretch vibration was probed in the latter, resulting in a red-shifted spectrum). Some first experimental measurements addressed to the water SFG spectrum at the graphene interface have recently been reported. ${ }^{5}$ The overall SFG intensity was measured; in the absence of phase information, the sign of $\chi^{(2)}$ is thus not known. The measured spectrum does exhibit low- and high-frequency bands as in Fig 3a, although the dangling $\mathrm{OH}$ band's intensity seems to be quite a bit smaller than in both our calculations and those of ref. 39. However, the experimental setup does not use the isotopic mixture studied in our simulations, and in addition employs a graphene sheet on a polar aluminum oxide (sapphire) support layer, whose electrostatic interactions with water might be responsible for the observed difference.

We now consider how vibrational SFG spectroscopy can probe the structural rearrangement within the interfacial layer that is induced by an electrode potential. Here we focus on the negatively charged interface, where the rearrangement is more pronounced. The SFG spectra shown in Fig 3b exhibit a dramatic change with increasingly negative potentials.

The first important change is the simultaneous growth and redshift of the high-frequency, dangling $\mathrm{OH}$ band. Both are due to the growing negative charges on the carbon atoms. The latter stabilize $\mathrm{OH}$ groups oriented towards the interface (as visible in Fig 1b), thus increasing their population and the band amplitude; further, the strengthening of the OH-graphene interaction redshifts the $\mathrm{OH}$ vibrational frequency, as found for a stronger hydrogen-bond acceptor.

The second major SFG spectra change in Fig $3 \mathrm{~b}$ is the spectacular growth of the $\simeq 3400$ 


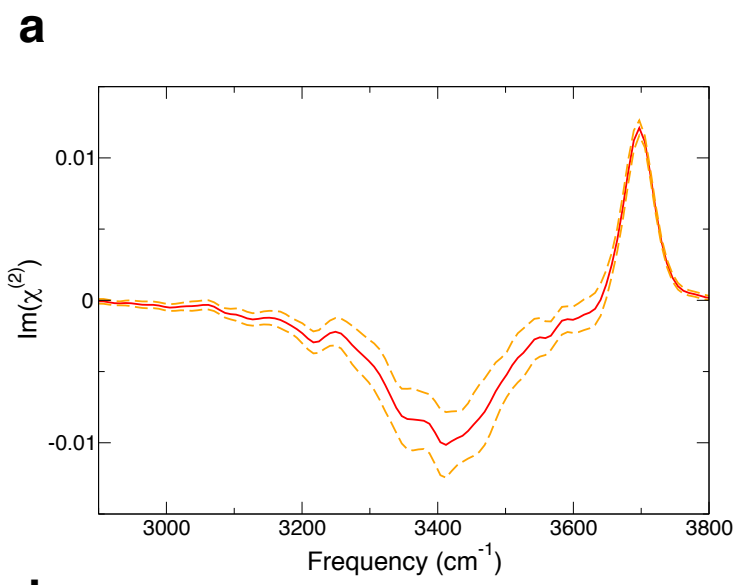

\section{b}

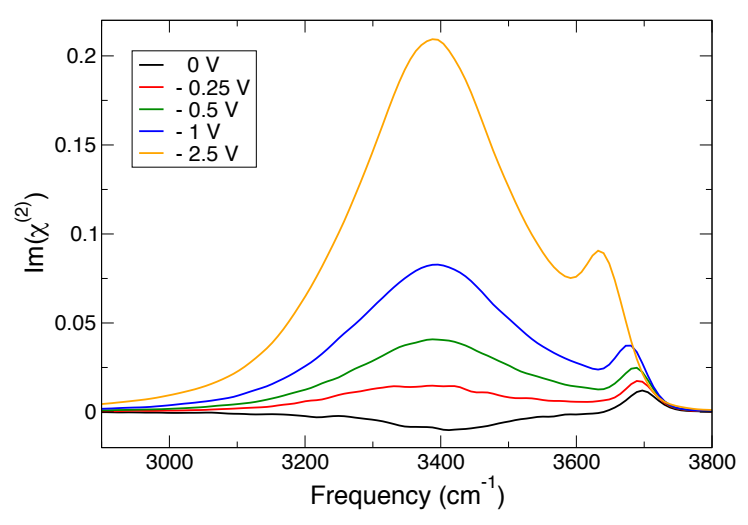

Figure 3: Calculated phase resolved vibrational SFG spectra of water (after convolution with a $10 \mathrm{~cm}^{-1}$ Gaussian), a) at $0 \mathrm{~V}$ with the average spectrum (red) and the $95 \%$ confidence interval (orange dashes), b) at the negatively charged graphene interfaces for a series of applied potentials (the calculated $3400 \mathrm{~cm}^{-1}$ peak height is underestimated due to the cutoff applied to the $\chi^{(3)}$ contribution beyond half the simulation box length).

$\mathrm{cm}^{-1}$ hydrogen-bonded band. This band is negative at $0 \mathrm{~V}$ and becomes positive for increasingly negative electrode potentials, growing to such extent that it greatly surpasses the amplitude of the dangling $\mathrm{OH}$ band for the most negative potentials investigated. But while the band's amplitude dramatically changes with the electrode potential, its central frequency remains unchanged. At negative potentials, the positive sign of $\operatorname{Im}\left[\chi^{(2)}\right]$ implies that the $\mathrm{OH}$ groups causing the signal are pointing towards graphene, while at the same time the essentially constant $\simeq 3400 \mathrm{~cm}^{-1}$ frequency shows that these groups are donating strong hydrogen-bonds. These two features imply that these $\mathrm{OH}$ groups cannot be in the first layer 
next to the graphene interface. The question then is: where are they located?

This query brings us to a long-standing question in SFG spectroscopy: what is the depth of the interface that is experimentally probed ${ }^{40}$ ? Since SFG specifically probes water molecules residing in a non-centrosymmetric environment, answering this question amounts to determining how far the interface-induced perturbation extends. In the case of the air/water interface, it was established that only the two top-most layers contribute to the SFG spectrum, ${ }^{41}$ but it was shown that for e.g. surfactant/water interfaces that the probed depth changes with vibrational frequency and can have a greater extent. ${ }^{42}$ Accordingly, we undertook to directly calculate the SFG spectrum's contributions from the successive water layers when different electrode potentials are applied.

We first consider the $0 \mathrm{~V}$ spectrum as an essential reference. Figure 4a shows the depthdependent spectrum along the distance $z_{H}$ from the water $\mathrm{OH}$ group hydrogen atom to the graphene plane. This figure allows us to considerably refine and deepen the molecular interpretation of the SFG spectrum in Fig 3a. First, it confirms that the high-frequency $\simeq$ $3700 \mathrm{~cm}^{-1}$ peak indeed comes almost exclusively from first-layer $\mathrm{OH}$ groups pointing towards the interface, as shown by the short $z_{H} \simeq 2.7 \AA$ distance where this peak appears. Second, and in contrast, the $\chi^{(2)}$ signal at lower frequencies includes not only contributions from the first layer but also from the second layer. This latter region requires a more detailed discussion, which we initiate with Fig 4a, which shows an alternation of positive and negative contributions for growing $z_{H}$, due to the two $\mathrm{OH}$ groups carried by the water molecules in each layer. Here it is useful to recall that the density profile maxima in Fig 1a provide the water oxygen atoms' positions in each layer, and the first two peaks are successively located at $3.5 \AA$ and $6.7 \AA$ from the interface at $0 \mathrm{~V}$. We can then see that the negative band at $\simeq 3400 \mathrm{~cm}^{-1}$ results from a series of contributions - two for each layer - with an important cancellation of effects. For the first layer contributions, there is: i) a positive one at $z_{H} \simeq 3.3 \AA$ arising from $\mathrm{OH}$ groups having undergone a large-amplitude librational motion, which brings them from their tangent equilibrium hydrogen-bonded arrangement 
into configurations pointing towards the surface, and ii) a negative one from $\mathrm{OH}$ groups pointing towards the bulk $\left(z_{H} \simeq 4.3 \AA\right)$; this is the dominant term in the spectrum's negative band. (We pause to note that contribution ii) is centered at a higher frequency than in the resulting SFG spectrum $\left(3500 \mathrm{~cm}^{-1}\right.$ vs $\left.3400 \mathrm{~cm}^{-1}\right)$ and that the apparent frequency shift results from a more pronounced cancellation with term i) at higher frequency.) For the second layer contributions, there is iii) a positive one from $\mathrm{OH}$ groups pointing towards the interface $\left(z_{H} \simeq 5.7 \AA\right)$ and finally iv) a negative one from $\mathrm{OH}$ groups pointing away from the interface $\left(z_{H} \simeq 7.0 \AA\right)$. No significant SFG contribution was found beyond the second layer at $0 \mathrm{~V}$.

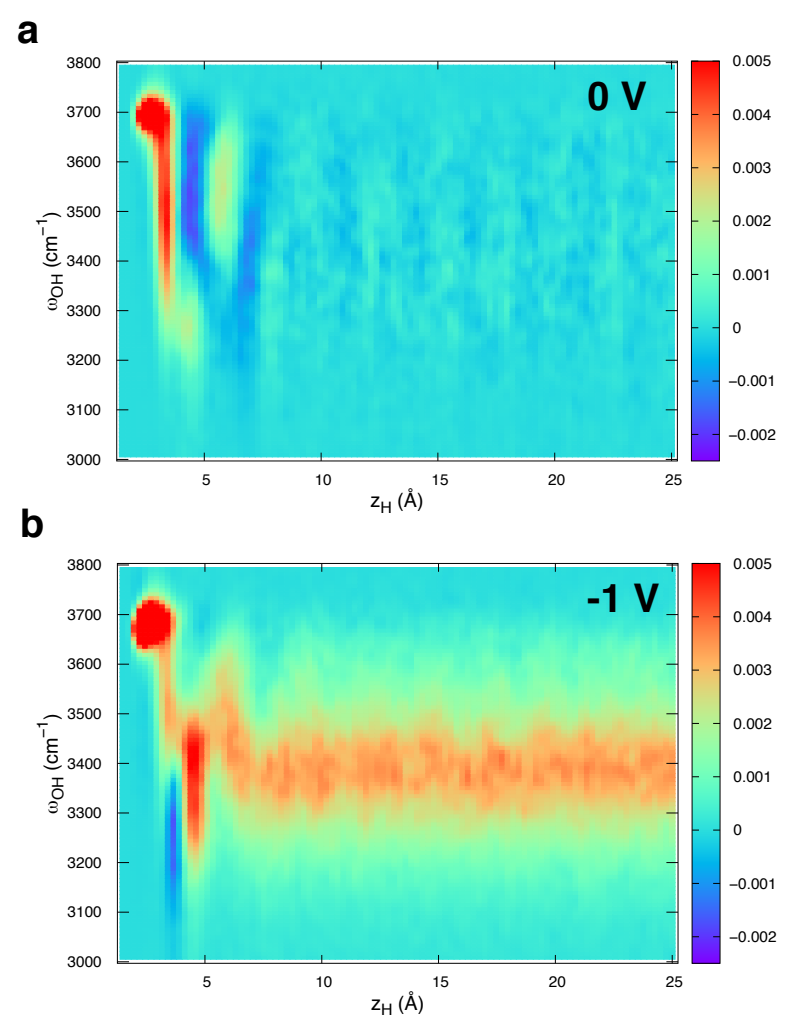

Figure 4: Depth-dependent contributions to the SFG spectrum along the distance $z_{H}$ between the water hydrogen atom and the graphene interface a) at $0 \mathrm{~V}$ and $\mathrm{b}$ ) at $-1 \mathrm{~V}$. At each distance, the eq $2 \chi_{p q r}^{(2)}(\omega)$ calculation is restricted to water $\mathrm{OH}$ groups with a specific $z_{H}$ value at $t=0$.

We now contrast these zero potential results with the situation at $-1 \mathrm{~V}$, shown in Fig 4b. First, the enhancement and redshift of the high-frequency peak is now very clear, and 
remains exclusively due to first-layer $\mathrm{OH}$ groups pointing towards the interface. However, the lower-frequency part of the spectrum changes dramatically with respect to the $0 \mathrm{~V}$ case. First, the first two layers' contributions are now strongly affected; as shown in Fig 1b's structural analysis, the number of $\mathrm{OH}$ groups pointing towards the bulk decreases - thus decreasing their negative SFG spectrum contribution - while most interfacial OH groups remain approximately tangent to the graphene electrode and bring a small signal with the ssp polarization. However, the most important change is the presence of contributions not only from the closest two water layers but also from water molecules much deeper than those layers. We see that the contribution to the SFG spectrum continues past them, becoming approximately constant for distances greater than $\simeq 7 \AA$. This long-range signal arises from the static electric field created between the graphene sheets and induces a so-called $\chi^{(3)}$ signal.

The importance of the $\chi^{(3)}$ contribution at charged interfaces has already been recognized and abundantly discussed in the literature (see e.g. refs 31,40,43-49). However, in most studied systems so far - including e.g. charged silica/water and lipid/water interfaces - the surface charges are changed by modifying the $\mathrm{pH}$ or increasing the concentration in ions ${ }^{40}$ or charged surfactants, ${ }^{48,49}$ all of which induce a modification in the interface chemistry. The present electrified graphene/water interface thus offers an interesting platform to study long-range $\chi^{(3)}$ effects, since the surface charge can be independently set by changing the applied electrode potential. On-going work is being devoted to analysis of this contribution.

Our study has two key features. First, it has revelead that an electrode electric potential has a profound impact on the water layer in contact with a graphene sheet. The algebraic sign of the applied potential is found to have opposite effects on the interfacial water dynamics: while a positive potential slows down the reorientational and translational dynamics of water, a negative potential accelerates the interfacial water dynamics. The general features of these dynamics can be comprehended via the jump picture for water hydrogen bond exchange; this aspect will be pursued in detail elsewhere. The second key feature concerns vibrational 
SFG spectroscopic applicability at the interface. We also first show that the arrangement of water molecules at the graphene interface at $0 \mathrm{~V}$ is very similar to that found at the air/water interface, and that SFG spectroscopy can specifically probe this interface. But when non-vanishing electrode potentials are applied, the resulting static electric field induces a long-range response, which makes the SFG spectra interpretation a greater challenge, one which we will address in the future.

\section{Supporting Information Available}

The following files are available free of charge. Details of system preparation, density profiles, and SFG spectra calculations.

\section{References}

(1) Ambrosi, A.; Chua, C.; Bonanni, A.; Pumera, M. Electrochemistry of graphene and related materials. Chem Rev 2014, 114, 7150-7188.

(2) Striolo, A.; Michaelides, A.; Joly, L. The Carbon-Water Interface: Modeling Challenges and Opportunities for the Water-Energy Nexus. Annu Rev Chem Biomol Eng 2016, 7, 533-556.

(3) Lui, C.; Liu, L.; Mak, K.; Flynn, G.; Heinz, T. Ultraflat graphene. Nature 2009, 462, 339-341.

(4) Eda, G.; Mattevi, C.; Yamaguchi, H.; Kim, H.; Chhowalla, M. Insulator to Semimetal Transition in Graphene Oxide. J Phys Chem C 2009, 113, 15768-15771.

(5) Singla, S.; Anim-Danso, E.; Islam, A. E.; Ngo, Y.; Kim, S. S.; Naik, R. R.; Dhinojwala, A. Insight on Structure of Water and Ice Next to Graphene Using SurfaceSensitive Spectroscopy. ACS Nano 2017, 11, 4899-4906. 
(6) Schmidt, J.; Roberts, S.; Loparo, J.; Tokmakoff, A.; Fayer, M.; Skinner, J. Are water simulation models consistent with steady-state and ultrafast vibrational spectroscopy experiments? Chem Phys 2007, 341, 143-157.

(7) Dreier, L. B.; Liu, Z.; Narita, A.; van Zadel, M.-J.; Müllen, K.; Tielrooij, K.-J.; Backus, E. H. G.; Bonn, M. Surface-Specific Spectroscopy of Water at a Potentiostatically Controlled Supported Graphene Monolayer. J Phys Chem C 2019, asap.

(8) Rana, M.; Chandra, A. Ab initio and classical molecular dynamics studies of the structural and dynamical behavior of water near a hydrophobic graphene sheet. J Chem Phys 2013, 138, 204702.

(9) Bie, Y.; Horng, J.; Shi, Z.; Ju, L.; Zhou, Q.; Zettl, A.; Yu, D.; Wang, F. Vibrational spectroscopy at electrolyte/electrode interfaces with graphene gratings. Nat Commun 2015, 6, 7593 .

(10) Siepmann, J. I.; Sprik, M. Influence of surface topology and electrostatic potential on water/electrode systems. J Chem Phys 1995, 102, 511.

(11) Reed, S.; Lanning, O.; Madden, P. Electrochemical interface between an ionic liquid and a model metallic electrode. J Chem Phys 2007, 126, 084704.

(12) Willard, A. P.; Reed, S. K.; Madden, P. A.; Chandler, D. Water at an electrochemical interface - a simulation study. Faraday Discuss. 2009, 141, 423-441.

(13) Merlet, C.; Péan, C.; Rotenberg, B.; Madden, P.; Simon, P.; Salanne, M. Simulating Supercapacitors: Can We Model Electrodes As Constant Charge Surfaces. J Phys Chem Lett 2013, 4, 264-268.

(14) Wang, Z.; Yang, Y.; Olmsted, D.; Asta, M.; Laird, B. Evaluation of the constant potential method in simulating electric double-layer capacitors. J Chem Phys 2014, $141,184102$. 
(15) Plimpton, S. Fast parallel algorithms for short-range molecular dynamics. J Comp Phys $1994,117,1-19$.

(16) Du, Q.; Freysz, E.; Shen, Y. R. Surface vibrational spectroscopic studies of hydrogen bonding and hydrophobicity. Science 1994, 264, 826-828.

(17) Stirnemann, G.; Rossky, P. J.; Hynes, J. T.; Laage, D. Water Reorientation, HydrogenBond Dynamics and 2D-IR Spectroscopy next to an Extended Hydrophobic Surface. Faraday Discuss 2010, 146, 263-281.

(18) Xiao, S.; Figge, F.; Stirnemann, G.; Laage, D.; McGuire, J. A. Orientational Dynamics of Water at an Extended Hydrophobic Interface. J Am Chem Soc 2016, 138, 5551-5560.

(19) Ho, T. A.; Striolo, A. Molecular dynamics simulation of the graphene-water interface: comparing water models. Mol Simulat 2014, 40, 1190-1200.

(20) Ruiz-Barragan, S.; Muñoz-Santiburcio, D.; Marx, D. Nanoconfined Water within Graphene Slit Pores Adopts Distinct Confinement-Dependent Regimes. J Phys Chem Lett 2019, 10, 329-334.

(21) Subasinghege Don, V.; David, R.; Du, P.; Milet, A.; Kumar, R. Interfacial Water at Graphene Oxide Surface: Ordered or Disordered. J Phys Chem B 2019, 123, 16361649.

(22) Rajamani, S.; Ghosh, T.; Garde, S. Size dependent ion hydration, its asymmetry, and convergence to macroscopic behavior. J Chem Phys 2004, 120, 4457-4466.

(23) Laage, D.; Stirnemann, G.; Sterpone, F.; Rey, R.; Hynes, J. T. Reorientation and Allied Dynamics in Water and Aqueous Solutions. Annu Rev Phys Chem 2011, 62, 395-416.

(24) Laage, D.; Hynes, J. T. A molecular jump mechanism of water reorientation. Science 2006, 311, 832-835. 
(25) Fogarty, A.; Duboué-Dijon, E.; Sterpone, F.; Hynes, J.; Laage, D. Biomolecular hydration dynamics: a jump model perspective. Chem Soc Rev 2013, 42, 5672-5683.

(26) Wilkins, D. M.; Manolopoulos, D. E.; Pipolo, S.; Laage, D.; Hynes, J. T. Nuclear Quantum Effects in Water Reorientation and Hydrogen-Bond Dynamics. J Phys Chem Lett 2017, 8, 2602-2607.

(27) Stirnemann, G.; Castrillón, S.; Hynes, J.; Rossky, P.; Debenedetti, P.; Laage, D. Nonmonotonic dependence of water reorientation dynamics on surface hydrophilicity: competing effects of the hydration structure and hydrogen-bond strength. Phys Chem Chem Phys 2011, 13, 19911-19917.

(28) Laage, D.; Elsaesser, T.; Hynes, J. Water Dynamics in the Hydration Shells of Biomolecules. Chem Rev 2017, 117, 10694-10725.

(29) Shen, Y. R.; Ostroverkhov, V. Sum-frequency vibrational spectroscopy on water interfaces: polar orientation of water molecules at interfaces. Chem Rev 2006, 106, 11401154.

(30) Ishiyama, T.; Imamura, T.; Morita, A. Theoretical Studies of Structures and Vibrational Sum Frequency Generation Spectra at Aqueous Interfaces. Chem Rev 2014, 114, $8447-8470$.

(31) Nihonyanagi, S.; Yamaguchi, S.; Tahara, T. Ultrafast Dynamics at Water Interfaces Studied by Vibrational Sum Frequency Generation Spectroscopy. Chem Rev 2017, $117,10665-10693$.

(32) Zheng, W.; Tadjeddine, A. Adsorption processes and structure of water molecules on Pt(110) electrodes in perchloric solutions. J Chem Phys 2003, 119, 13096-13099.

(33) Nihonyanagi, S.; Ye, S.; Uosaki, K.; Dreesen, L.; Humbert, C.; Thiry, P.; Peremans, A. 
Potential-dependent structure of the interfacial water on the gold electrode. Surf Sci 2004, 573, 11-16.

(34) Schultz, Z. D.; Shaw, S. K.; Gewirth, A. A. Potential Dependent Organization of Water at the Electrified Metal-Liquid Interface. J Am Chem Soc 2005, 127, 15916-15922.

(35) Morita, A.; Hynes, J. T. A Theoretical Analysis of the Sum Frequency Generation Spectrum of the Water Surface. II. Time-Dependent Approach. J Phys Chem B 2002, $106,673-685$.

(36) Auer, B.; Skinner, J. Vibrational sum-frequency spectroscopy of the liquid/vapor interface for dilute HOD in D(2)O. J Chem Phys 2008, 129, 214705.

(37) Byrnes, S. J.; Geissler, P. L.; Shen, Y. Ambiguities in surface nonlinear spectroscopy calculations. Chem Phys Lett 2011, 516, 115-124.

(38) Ni, Y.; Skinner, J. Communication: Vibrational sum-frequency spectrum of the airwater interface, revisited. J Chem Phys 2016, 145, 031103.

(39) Ohto, T.; Tada, H.; Nagata, Y. Structure and dynamics of water at water-graphene and water-hexagonal boron-nitride sheet interfaces revealed by ab initio sum-frequency generation spectroscopy. Phys Chem Chem Phys 2018, 20, 12979-12985.

(40) Gonella, G.; Lütgebaucks, C.; de Beer, A. G. F.; Roke, S. Second Harmonic and SumFrequency Generation from Aqueous Interfaces Is Modulated by Interference. J Phys Chem C 2016, 120, 9165-9173.

(41) Morita, A.; Hynes, J. T. A theoretical analysis of the sum frequency generation spectrum of the water surface. Chem Phys 2000, 258, 371-390.

(42) Roy, S.; Gruenbaum, S.; Skinner, J. Theoretical vibrational sum-frequency generation spectroscopy of water near lipid and surfactant monolayer interfaces. J Chem Phys 2014, 141, 18C502. 
(43) Ong, S.; Zhao, X.; Eisenthal, K. B. Polarization of water molecules at a charged interface: second harmonic studies of the silica/water interface. Chem Phys Lett 1992, 191, $327-335$.

(44) de Beer, A. G.; Campen, R. K.; Roke, S. Separating surface structure and surface charge with second-harmonic and sum-frequency scattering. Phys Rev B 2010, 82, 235431.

(45) Ohno, P.; Wang, H.; Geiger, F. Second-order spectral lineshapes from charged interfaces. Nat Commun 2017, 8, 1032.

(46) Joutsuka, T.; Hirano, T.; Sprik, M.; Morita, A. Effects of third-order susceptibility in sum frequency generation spectra: a molecular dynamics study in liquid water. Phys Chem Chem Phys 2018, 20, 3040-3053.

(47) Hore, D. K.; Tyrode, E. Probing Charged Aqueous Interfaces Near Critical Angles: Effect of Varying Coherence Length. J Phys Chem C 2019, 123, 16911-16920.

(48) Wen, Y.; Zha, S.; Liu, X.; Yang, S.; Guo, P.; Shi, G.; Fang, H.; Shen, Y.; Tian, C. Unveiling Microscopic Structures of Charged Water Interfaces by Surface-Specific Vibrational Spectroscopy. Phys Rev Lett 2016, 116, 016101.

(49) Dreier, L.; Nagata, Y.; Lutz, H.; Gonella, G.; Hunger, J.; Backus, E.; Bonn, M. Saturation of charge-induced water alignment at model membrane surfaces. Sci Adv 2018, 4, eaap7415. 


\title{
Supplementary Information
}

\section{Water structure, dynamics and sum-frequency generation spectra at electrified graphene interfaces}

\author{
Yiwei Zhang, ${ }^{\dagger}$ Hilton B. de Aguiar, ${ }^{\ddagger}$ James T. Hynes, ${ }^{\dagger}$, and Damien Laage ${ }^{*} \dagger$ \\ $\dagger$ †ASTEUR, Department of Chemistry, École normale supérieure, PSL University, \\ Sorbonne Université, CNRS, 75005 Paris, France \\ $\ddagger$ Département de Physique, École normale supérieure, PSL University, CNRS, 24 rue \\ Lhomond, 75005 Paris, France \\ \Department of Chemistry, University of Colorado, Boulder, CO 80309, USA \\ E-mail: damien.laage@ens.fr
}




\section{System preparation}

The graphene sheets are constructed from an ideal hexagonal lattice with a $1.42 \AA$ distance between nearest neighbor carbon atoms. ${ }^{1}$ The number of water molecules is determined iteratively with an insertion/deletion procedure and a series of short molecular dynamics simulation runs, until the density in the middle of the box reaches the experimental liquid water density of $0.997 \mathrm{~g} / \mathrm{cm}^{3}$ (see Fig. 1a).

\section{Oxygen and Hydrogen density profiles}

Figure S1 shows the contributions of oxygen and hydrogen atoms to the overall water density (Fig. 1a).

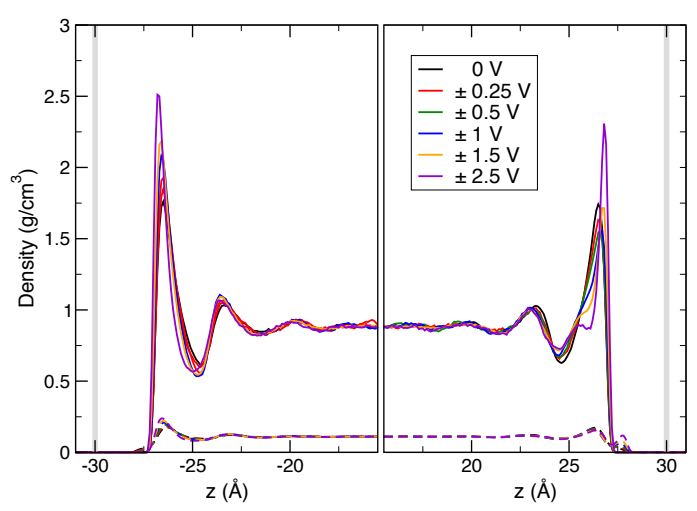

Figure S1: Oxygen (solid lines) and hydrogen (dashes) density profiles.

\section{Fraction of dangling $\mathrm{OH}$ groups}

Different geometric criteria have been suggested to define dangling water $\mathrm{OH}$ groups at an aqueous interface (see e.g. refs $^{2,3}$ ). Here we define as dangling the water $\mathrm{OH}$ groups whose oxygen atom lies within the first interfacial layer (determined from the density profile) and whose angle with the graphene normal vector is less than $40^{\circ}$. The fraction of interfacial dangling $\mathrm{OH}$ groups can thus be determined by integrating the distribution in Fig. 1b up to $40^{\circ}$. At $0 \mathrm{~V}$, the fraction of dangling groups among the total population of water $\mathrm{OH}$ groups within the interfacial layer is $\simeq 5 \%$. This can be compared to the fraction that was determined in SFG experiments at the air/water interface. ${ }^{4}$ These measurements had 
estimated that $20 \%$ of surface water molecules have a free $\mathrm{OH}$ group. If we now consider the population of $\mathrm{OH}$ groups at the interface, it suggests that $10 \%$ of interfacial water $\mathrm{OH}$ groups are in a dangling situation at the air/water interface, thus slightly more than at the $0 \mathrm{~V}$ graphene/water interface.

\section{OH stretch frequency map and SFG spectra calculations}

The water $\mathrm{OH}$ stretch vibrational frequency is calculated at each simulation time step using the empirical frequency map developed by Skinner and coworkers. ${ }^{5}$ We briefly summarize its main features. This spectroscopic map was parameterized on a series of water clusters. For each cluster, the $\mathrm{OH}$ stretch vibrational potential was determined and the quantum vibrational energy levels were determined. The resulting 0-1 energy gap was shown to exhibit a very good correlation with the local electric field projected along the $\mathrm{OH}$ bond as obtained from the simulation. This yields an empirical map connecting the local electric field determined in the MD simulation with a rigid water model and the vibrational frequency. Similar maps were developed for other quantities including the transition dipole and the polarizability tensor. These quantities are therefore affected by fluctuations in the local electric field and are able to reproduce the well-established $\mathrm{OH}$ frequency shift with the hydrogen-bonding strength. ${ }^{6}$

This frequency map was originally designed based on clusters taken from bulk liquid water simulations, but it was shown to successfully describe the water $\mathrm{OH}$ stretch properties in a series of other aqueous environments, including aqueous solutions (neat water, ionic solutions, aqueous solutions of amphiphilic molecules) and a wide gamut of aqueous interfaces (air/water, water/phospholipids, reverse micelle interfaces) (see ref. ${ }^{5}$ and references therein), which attests to its wide applicability. We therefore expect this frequency map to be well applicable to water/graphene interfaces, since graphene-water interactions are known to remain weak, in contrast with typical aromatic systems like benzene. The weakness of watergraphene interactions is supported by several ab initio molecular dynamics simulations. ${ }^{7,8}$

In neat $\mathrm{H}_{2} \mathrm{O}$, the vibrational spectra in the $\mathrm{OH}$ stretch region include important contri- 
butions due to the intra- and inter-molecular coupling between $\mathrm{OH}$ vibrations. A discussion of these terms for the air/water SFG spectrum can be found in ref. ${ }^{9}$ In order to avoid the complications due to these coupling terms and focus on a single bond vibration, isotopic mixtures of a dilute $\mathrm{HOD}$ in $\mathrm{H}_{2} \mathrm{O}$ or $\mathrm{D}_{2} \mathrm{O}$ are commonly employed. It was shown ${ }^{10}$ that simulations of e.g. pure $\mathrm{D}_{2} \mathrm{O}$ and $\mathrm{HOD}$ in $\mathrm{D}_{2} \mathrm{O}$ give very similar results for the vibrational spectra; we therefore follow the approach that was successfully used by Skinner and coworkers to calculate SFG spectra, ${ }^{11}$ and use our pure $\mathrm{H}_{2} \mathrm{O}$ trajectory, and treat each $\mathrm{OH}$ group as an independent $\mathrm{OH}$ oscillator to calculate the SFG spectrum.

\section{References}

(1) Cooper, D. R.; D'Anjou, B.; Ghattamaneni, N.; Harack, B.; Hilke, M.; Horth, A.; Majlis, N.; Massicotte, M.; Vandsburger, L.; Whiteway, E. et al. Experimental Review of Graphene. ISRN Condensed Matter Physics 2012, 2012, 1-56.

(2) Xiao, S.; Figge, F.; Stirnemann, G.; Laage, D.; McGuire, J. A. Orientational Dynamics of Water at an Extended Hydrophobic Interface. J Am Chem Soc 2016, 138, 5551-5560.

(3) Tang, F.; Ohto, T.; Hasegawa, T.; Xie, W.; Xu, L.; Bonn, M.; Nagata, Y. Definition of Free O-H Groups of Water at the Air-Water Interface. J Chem Theory Comput 2018, $14,357-364$.

(4) Du,; Superfine,; Freysz,; Shen, Vibrational spectroscopy of water at the vapor/water interface. Phys Rev Lett 1993, 70, 2313-2316.

(5) Gruenbaum, S. M.; Tainter, C. J.; Shi, L.; Ni, Y.; Skinner, J. L. Robustness of Frequency, Transition Dipole, and Coupling Maps for Water Vibrational Spectroscopy. J Chem Theory Comput 2013, 9, 3109-3117.

(6) Pimentel, G.; A, M. The Hydrogen Bond; W.H. Freeman and Co.: San Francisco and London, 1960. 
(7) Rana, M.; Chandra, A. Ab initio and classical molecular dynamics studies of the structural and dynamical behavior of water near a hydrophobic graphene sheet. J Chem Phys 2013, 138, 204702.

(8) Ohto, T.; Tada, H.; Nagata, Y. Structure and dynamics of water at water-graphene and water-hexagonal boron-nitride sheet interfaces revealed by ab initio sum-frequency generation spectroscopy. Phys Chem Chem Phys 2018, 20, 12979-12985.

(9) Pieniazek, P. A.; Tainter, C. J.; Skinner, J. L. Interpretation of the water surface vibrational sum-frequency spectrum. J Chem Phys 2011, 135, 044701.

(10) Corcelli, S. A.; Lawrence, C. P.; Asbury, J. B.; Steinel, T.; Fayer, M. D.; Skinner, J. L. Spectral diffusion in a fluctuating charge model of water. J Chem Phys 2004, 121, 8897-8900.

(11) Auer, B.; Skinner, J. Vibrational sum-frequency spectroscopy of the liquid/vapor interface for dilute HOD in D(2)O. J Chem Phys 2008, 129, 214705. 